\title{
The USP7 Inhibitor P5091 Induces Cell Death in Ovarian Cancers with Different P53 Status
}

\author{
Mengying Wang ${ }^{\mathrm{a}, \mathrm{b}} \quad$ Yayun Zhang ${ }^{\mathrm{a}} \quad$ Taishu Wang ${ }^{\mathrm{a}} \quad$ Jinrui Zhang ${ }^{\mathrm{a}} \quad$ Zhu Zhou ${ }^{\mathrm{b}}$ \\ Yan Sun ${ }^{\mathrm{b}}$ Shanshan Wang ${ }^{\mathrm{a}}$ Yulin Shi ${ }^{\mathrm{a}}$ Xuelin Luan ${ }^{\mathrm{a}} \quad$ Yingqiu Zhang ${ }^{\mathrm{a}}$ \\ Yifei Wang ${ }^{b}$ Yang Wang ${ }^{a}$ Zhongwen Zou ${ }^{a, b} \quad$ Lan Kang $^{a} \quad$ Han Liu ${ }^{a, c}$ \\ aThe Second Affiliated Hospital, Institute of Cancer Stem Cell, Dalian Medical University, Dalian, \\ bDepartment of Gynecology, Second Affiliated Hospital, Dalian Medical University, Dalian, 'Cancer \\ Biotherapy \& Translational Medicine Center of Liaoning Province, Dalian Medical University, Dalian, \\ China
}

\author{
Key Words \\ Usp7 • P5091 • Ovarian cancer • P53 • Apoptosis • Targeted therapy
}

\begin{abstract}
Background/Aims: Ovarian cancer is often diagnosed at later stages with poor prognosis. Recent studies have associated the expression of deubiquitylase USP7 with the survival of ovarian cancers. Being a cysteine protease, USP7 could become a target for pharmacological intervention. Therefore, in this study, we assessed the influence of its inhibitor P5091 on ovarian cancer cells. Methods: Ovarian cancer cells were treated with P5091, and cell proliferation was measured with MTT assay; cell morphology was inspected under a phase-contrast microscope; cell cycle and cell death were examined by flow cytometry. To gain mechanistic insights into its effects, immunoblotting was performed to detect USP7, HDM2, p53, p21, apoptosis and autophagy related proteins. Results: P5091 effectively suppressed the growth of ovarian cancer cells, caused cell cycle blockage, and induced necrosis and apoptosis with more severe phenotypes observed in HeyA8 cells with wild-type p53 than in OVCAR-8 cells with mutant p53. P5091 also prompted autophagy, with more efficient p62 degradation in HeyA8. Conclusion: P5091 shows efficacy in suppressing ovarian cancers harbouring wild-type and mutant p53. Its effects seemed to be enhanced by wild-type p53. The potency of this USP7 inhibitor also correlated with autophagy to some extent. Therefore, the pharmacological targeting of USP7 may serve as a potential therapeutic strategy and warrants further investigation.
\end{abstract}




\section{Introduction}

Ovarian cancer is a major type of cancer diagnosed in the field of gynecology. Deaths caused by ovarian cancer worldwide are on the rise, with estimations from approximately 100,000 cases in 1990 to more than 150, 000 cases in 2013 [1]. The majority of ovarian cancer cases are diagnosed at the later stages of the disease, and taxane and platinumbased chemotherapies are routinely adopted clinically [2, 3]. Approximately $3.5 \%$ and $3.2 \%$ of ovarian cancers harbour somatic mutations in BRCA1 and BRCA2, respectively; these mutations identify a subgroup of patients eligible for targeted therapy with the PARP inhibitor olaparib [4,5]. Another FDA-approved targeted therapy in the clinical management of ovarian cancer is the anti-angiogenesis agent Bevacizumab, which is a VEGF antibody [6, 7]. In view of these limited approved targeted therapies for ovarian cancer, many efforts have been made to search for novel intervention targets in order to improve patient survival [8].

The ubiquitin proteasome pathway is the major means by which cellular proteins are degraded via post-translational modifications with ubiquitin $[9,10]$. Deubiquitinating enzymes (DUBs) are a group of proteins that can remove ubiquitin moieties from substrates to reverse ubiquitination mediated cellular processes such as proteasomal degradation [11, 12]. Ubiquitin specific proteases (USPs) comprise the largest family of DUBs, with over 50 cysteine proteases; one of these, USP7, has been closely implicated in tumourigenesis and cancer progression $[13,14]$. It has recently been shown that the expression of USP7 correlates with lymph node metastasis and poor prognosis in epithelial ovarian cancers $[15,16]$. Based on these observations, in the present study we investigated whether the pharmacological inhibition of USP7 by its small molecule inhibitor P5091 can suppress ovarian cancer growth in vitro. We found that the USP7-inhibitor P5091 is capable of inducing cell death in ovarian cancers harbouring either wild-type or a mutant form of p53, suggesting that pharmacological targeting of USP7 in ovarian cancers may be a potential therapeutic strategy.

\section{Materials and Methods}

\section{Antibodies and other reagents}

Rabbit anti-USP7 (ab4080) and anti-p21 [EPR3993] (ab109199) antibodies were purchased from Abcam. Mouse anti-HDM2 (SMP14) and anti-p53 (D0-1, SC-126) antibodies were from Santa Cruz Biotechnology. Rabbit anti-Caspase-3 (9662), anti-BCL-2 (50E3) (2870), and anti-MCL-1 (D35A5) (5453) antibodies were obtained from Cell Signaling Technology. Mouse anti-GAPDH, rabbit anti-PARP1 (133711-AP), and mouse anti-Actin antibodies were from Proteintech (Wuhan, China). The USP7 inhibitor P5091 was purchased from Selleck.

\section{Cell culture}

Human ovarian cancer cell lines and normal cell line were incubated at $37^{\circ} \mathrm{C}$ in a humidified incubator (Thermo) with a $\mathrm{CO}_{2}$ concentration of 5\%. HeyA8 and OVCAR-8 cells were maintained in Dulbecco's modified Eagle's medium (DMEM, Gibco, USA), and SKOV3 cells were cultured in McCoy's 5A medium (Gibco, USA). The normal ovarian cell line T80 (a generous gift from Dr. Jin Q. Cheng, H. Lee Moffitt Cancer Center, US) was maintained in Dulbecco's modified Eagle's medium. All cells were cultured in the presence of $10 \%$ foetal bovine serum (Gibco, USA) and 1\% antibiotics (penicillin/streptomycin, Thermo Fisher Scientific).

\section{Cell proliferation assay}

The proliferation of ovarian cancer cells was determined using the 3-(4, 5-dimethylthiazol-2-yl)-2, 5-diphenyltetrazolium bromide tetrazolium (MTT) method. In brief, 4000 ovarian cancer cells per well were seeded in a 96-well plate and incubated overnight. The next day, cells were treated with the P5091 inhibitor or DMSO as control, for the indicated times. MTT was added, and cells were placed back into the incubator for 3 hours. Then, DMSO was added to dissolve formazan and absorbance was recorded at 570 nm with a spectrometer.

\section{KARGER}




\section{Cellular Physiology Cell Physiol Biochem 2017;43:1755-1766 and Biochemistry DOI: 10.1159/000484062 \begin{tabular}{l|l} 
O 2017 The Author(s). Published by S. Karger AG, Basel \\
wwww.karger.com/cpb
\end{tabular}}

Wang et al.: Targeting USP7 in Ovarian Cancer

\section{Western blotting}

Following PBS washes, ovarian cancer cells were lysed in RIPA buffer (1\% w/v Triton X-100, 0.1\% w/v SDS, 10 mM Tris- $\mathrm{HCl}$ pH 7.5, $150 \mathrm{mM} \mathrm{NaCl}, 1 \%$ sodium deoxycholate) supplemented with protease and phosphatase inhibitors. Cell lysates were centrifuged to remove aggregates and cell debris. Protein concentrations were measured using the Bradford assay. Western blotting was performed as previously described [17]. To summarise, proteins were subjected to SDS-PAGE analysis and blotted to nitrocellulose membranes (Merck Millipore). Membranes were blocked with 5\% non-fat milk in PBS at room temperature, before primary antibody incubation at $4 \stackrel{\circ}{\circ} \mathrm{C}$ overnight. Membranes were washed with PBS and incubated with corresponding secondary antibodies (LICOR $680 \mathrm{~nm}$ or $800 \mathrm{~nm}$ infrared labeled). Membranes were detected on a LICOR Odyssey scanner, and images were quantitated using Image Studio (Version 4.0) following the manufacturer's instructions.

\section{Fluorescence microscopy}

Hoechst 33342 staining was performed as previously described [18]. In brief, ovarian cancer cells were seeded onto 35-mm dishes and incubated under normal conditions overnight. The next day, cells were treated with the P5091 inhibitor (DMSO as control), before Hoechst 33342 was added to final a concentration of 5 $\mu \mathrm{g} / \mathrm{ml}$. Cells were replaced back in the incubator for another 10 minutes, and then staining was examined immediately using a reversed fluorescence microscope (Olympus, Japan) with Hoechst 33342 detected in the blue channel.

\section{Flow cytometry}

Human ovarian cancer cell lines (HeyA8 and OVCAR-8) were treated with $50 \mu \mathrm{M}$ of P5091 or DMSO as control. At indicated times, one million cells were collected from each condition. As previously described, in cell cycle assays harvested cells were washed with PBS and fixed in 70\% ice-cold ethanol, prior to staining with $50 \mu \mathrm{g} / \mathrm{ml}$ of propidium iodide (PI) $[19,20]$. In apoptotic assays, the cells were PBS-washed and processed for Annexin V and PI double-staining with an apoptosis assay kit (KeyGEN Biotech, China) as per the manufacturer's instruction. Stained cells were analysed with a bench-top flow cytometer (Accuri C6, BD Biosciences). Flow cytometry data were analysed with FlowJo (Version 7.6.1, LLC, USA).

\section{Statistics}

Experiments were conducted with 3 independent biological repeats in order to compare difference between groups. Experimental data are represented as the means \pm standard error of the mean (SEM). Statistical differences were examined by performing two-tailed Student's t-test in GraphPad Prism software (Version 5.01), and $\mathrm{p}<0.05$ was used as a cut-off for statistical significance.

\section{Results}

The USP7 inhibitor P5091 inhibits ovarian cancer cell proliferation

To assess the effect of P5091 on the propagation of ovarian cancer cells in vitro, a series of concentrations (5-50 $\mu \mathrm{M})$ was added into culture media of SKOV3, HeyA8, and OVCAR-8 cell lines. Cell proliferation was measured with MTT assays. Among the three cell lines, SKOV3 was the least sensitive, while HeyA8 growth was the most dramatically suppressed (Fig. 1A, B, C). At $50 \mu \mathrm{M}, \mathrm{P} 5091$ repressed SKOV3 growth to only 67\%, but inhibited the proliferation of HeyA8 and OVCAR-8 to an average of $22 \%$ and 33\%, respectively (Fig. 1A, B, C). Besides, we tested the impact of P5091 on the propagation of a normal ovarian cell line (T80). This USP7 inhibitor was also capable of inhibiting the growth of normal ovarian cells, but to a lesser extent compared to HeyA8 and OVCAR-8 (Fig. 1D). As p53 and HDM2 are the primary targets of USP7 and SKOV3 was p53-null, we picked HeyA8 and OVCAR-8 cells for further studies [21]. We treated both cell lines with P5091 and examined cell morphology under microscope. At 12 and 24 hours, control cells treated with DMSO propagated healthily, while the growth of P5091 treated HeyA8 and OVCAR-8 cells was remarkably repressed, with some cells exhibiting rounded shapes or even detaching from the culture dish, indicative of cell death (Fig. 1E and F). 


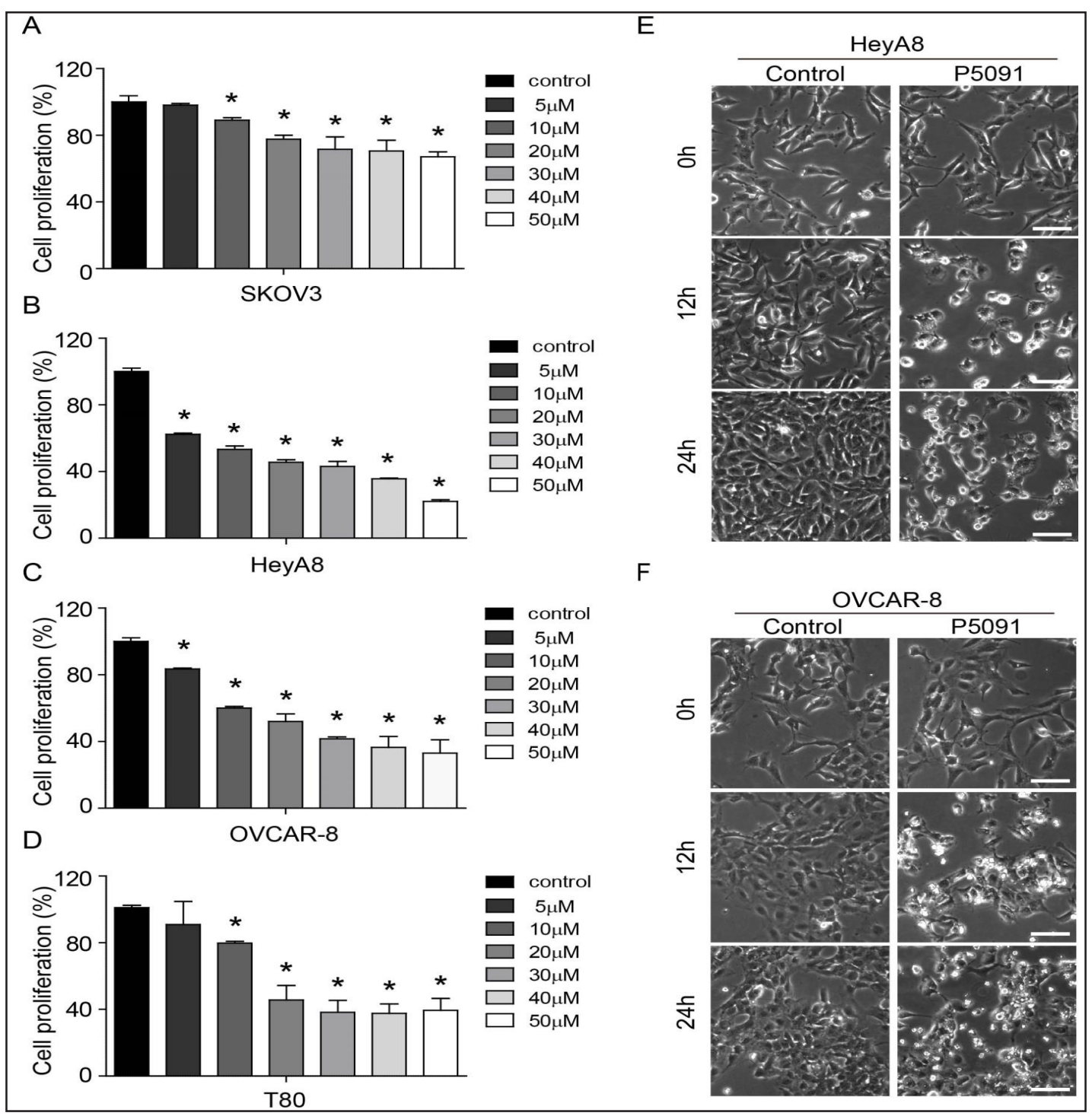

Fig. 1. The USP7 inhibitor P5091 suppresses ovarian cancer cell growth in vitro. Cultured ovarian cancer cells were treated with various concentrations of P5091 as indicated and cell proliferation was measured with MTT assays. A, B, C, and D represent SKOV3, HeyA8, OVCAR-8, and T80 cells, respectively. All error bars represent standard error of the mean $(n=3$, * represents $\mathrm{p}<0.05)$. E and F, HeyA8 and OVCAR-8 cells were treated with $50 \mu \mathrm{M}$ of P5091 for 12 and 24 hours before examination under a phase contrast microscope. Control cells were treated with DMSO. Representative micrographs are shown to illustrate morphology changes (scale bar $=100 \mu \mathrm{m})$.

In subsequent cell cycle analyses, P5091-treated HeyA8 and OVCAR-8 cells displayed reduced distribution in sub-G1 phase and an increased proportion in G2/M phase, with relatively stable $S$ phases (Fig. 2A and B). These results suggest that P5091 treatment led to cell cycle blockage in G2/M phase. Considering the evidence from cell morphology observations that P5091 might cause cell death, we stained P5091-treated HeyA8 and OVCAR-8 cells with the Hoechst 33342 dye, which penetrates dead cells more robustly. Compared with DMSOtreated control cells, Hoechst 33342 over-stained cells in the P5091-treated HeyA8 group were dramatically increased to $19.69 \%$ and $70.32 \%$ by 12 and 24 hours, respectively (Fig. 2C). Likewise, P5091 treatment also resulted in the elevation of Hoechst 33342 over-stained OVCAR- 8 cells to $4.66 \%$ and $22.01 \%$ at 12 and 24 hours, respectively (Fig. 2D).

\section{KARGER}




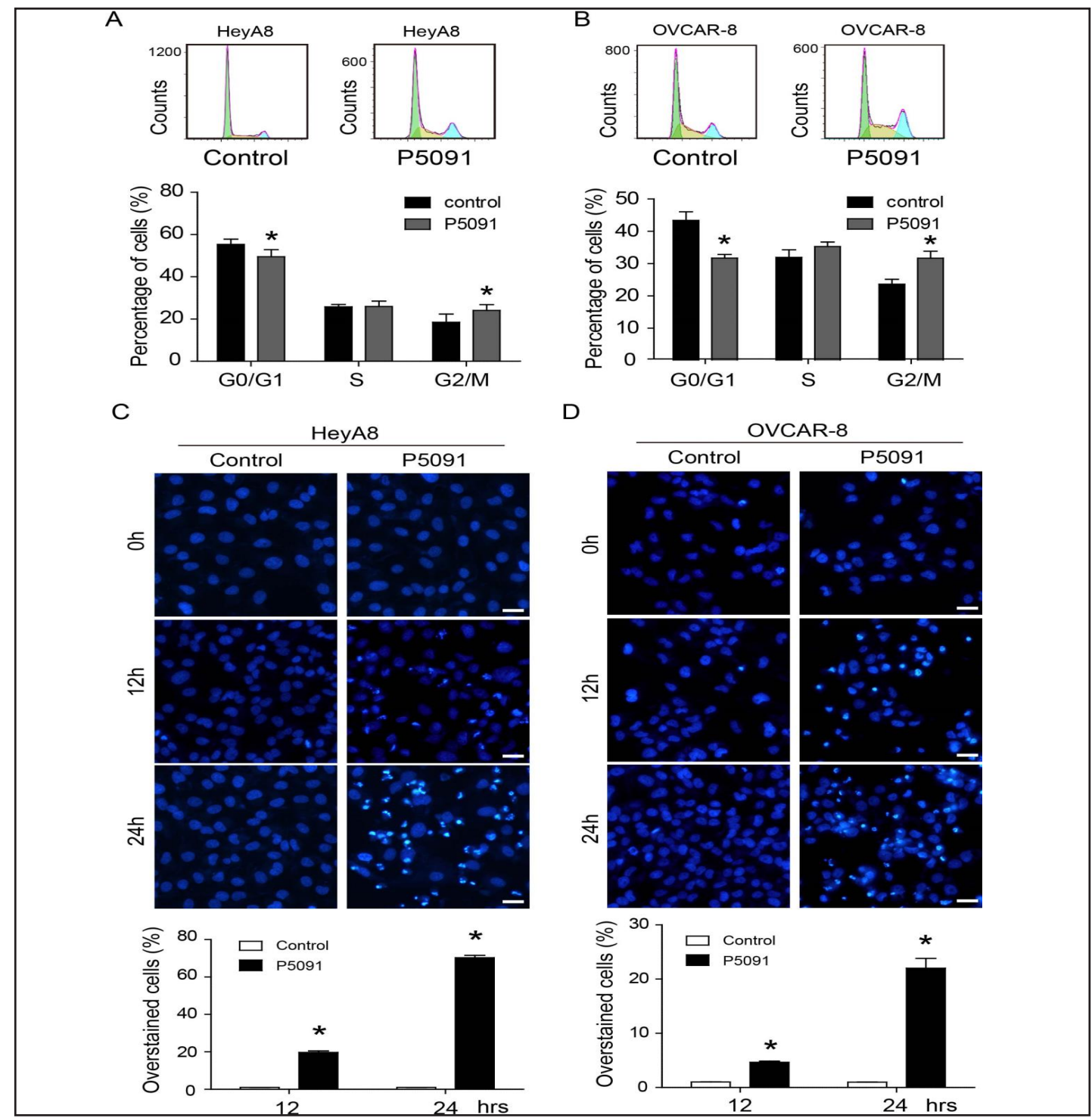

Fig. 2. P5091 induces cell cycle blockage and cell death. A and B, HeyA8 and OVCAR-8 cells were treated with P5091 at $50 \mu \mathrm{M}$ for 18 hours (or DMSO as control) and processed for cell cycle analysis. Above histograms show representative original data, and bar charts below demonstrate quantification data from 3 independent experiments. C and D, ovarian cancer cells were treated as mentioned above prior to Hoechst 33342 staining. Micrographs show representative images (scale bar $=20 \mu \mathrm{m}$ ). Bar charts show quantification results from 3 independent experiments. More than 200 cells were counted from random chosen views within each group. All error bars represent standard error of the mean $(n=3$, * represents $\mathrm{p}<0.05)$.

\section{P5091 induces cell death in ovarian cancer cells}

Since increased Hoechst 33342 staining primarily indicates plasma membrane damages and cell death, we performed flow cytometry with propidium iodide and Annexin V doublestaining to closely examine the features of P5091-triggered cell death and gain insights on the impact of P5091 on ovarian cancer cells. As shown in Fig. 3A, HeyA8 and OVCAR-8 cells treated with P5091 showed increased necrosis as evidenced by Annexin V negative and PI positive staining, which were $23.8 \%$ and $10.8 \%$ in HeyA8 and OVCAR-8, respectively. In addition, P5091 treatment in both ovarian cancer cell lines led to significant increases in apoptosis as revealed by enhanced Annexin V staining (23\% and $8.6 \%$ in HeyA8 and 
A

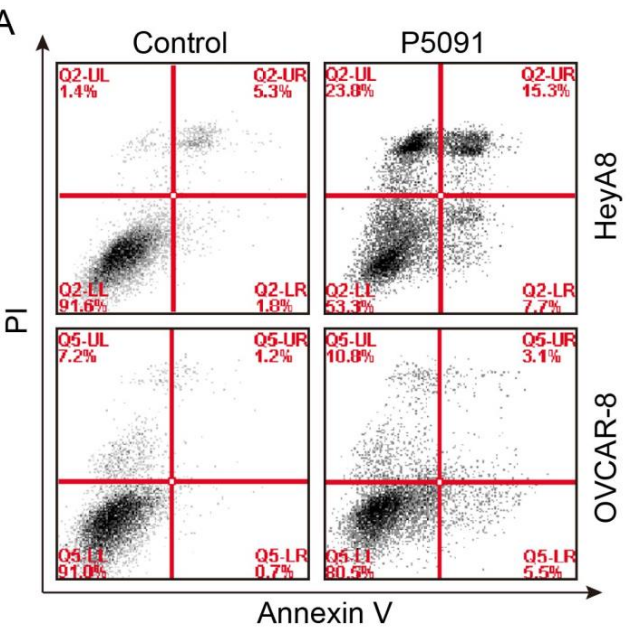

C

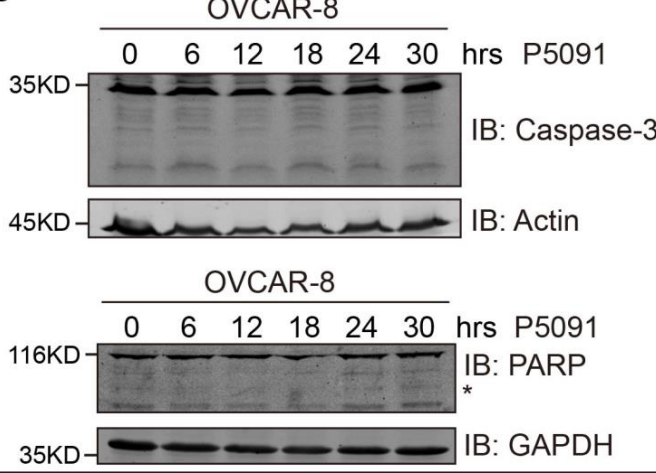

B

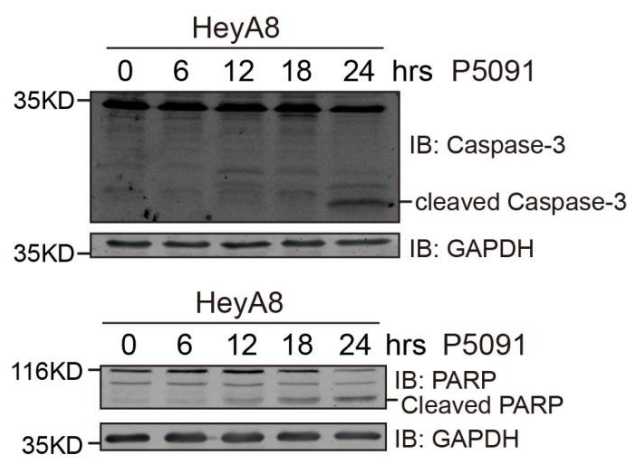

D

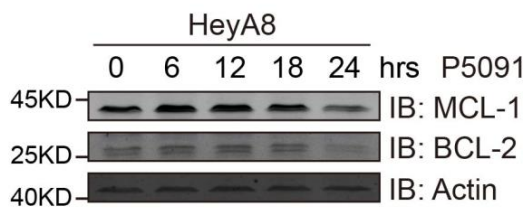

OVCAR-8

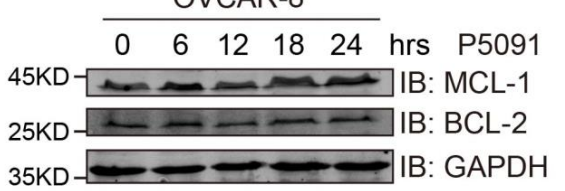

Fig. 3. Ovarian cancer cells undergo both necrosis and apoptosis following P5091 treatment. A, HeyA8 and OVCAR-8 cells were treated with $50 \mu \mathrm{M}$ of P5091 for 18 hours, while control cells were treated with DMSO. Cells were counted and double-labelled with PI and Annexin V before analysis using a flow cytometer. Scatter plots from representative data show increases in necrotic and apoptotic populations post P5091 addition. B and C, HeyA8 and OVCAR-8 cells were treated with $50 \mu \mathrm{M}$ of P5091 for indicated times and lysed to harvest proteins. Samples were resolved on SDS-PAGE gels and then transferred to nitrocellulose membranes for immunoblotting assays. Caspase- 3 and PARP were probed to detect apoptosis. GAPDH and Actin were probed to confirm equal loading. * denotes a faint band for cleaved PARP. D, samples from B and C were analysed by immunoblotting with MCL-1 and BCL-2 antibodies. GAPDH and Actin blots show equal loading.

OVCAR-8, respectively) (Fig. 3A). These results are consistent with data from Hoechst 33342 staining and cell proliferation assays, which revealed that P5091 exerted stronger effects on HeyA8 than OVCAR-8 cells. Nevertheless, it induced necrosis and apoptosis in both cell lines.

To confirm the pro-apoptotic effects of P5091 on ovarian cancer cells, we performed immunoblotting experiments to examine the expression of apoptosis-related markers. As illustrated in Fig. 3B and C, P5091 addition in HeyA8 cells resulted in the formation of cleaved forms of Caspase-3 and PARP indicating apoptosis. However, P5091-induced apoptosis appeared to be weaker in OVCAR-8 cells, as only a faint, cleaved band of PARP was detected after 30 hours. Furthermore, we examined the expression of anti-apoptotic proteins MCL-1 and BCL-2 in P5091-treated HeyA8 and OVCAR-8 cells. As shown in Fig. 3D, the levels of both MCL-1 and BCL-2 were reduced by P5091 in HeyA8 cells, while these anti-apoptotic proteins remained stable following P5091 treatment in OVCAR-8 cells. These data collectively suggest that OVCAR-8 cells are more resistant to P5091-induced cell death compared to HeyA8. 
A
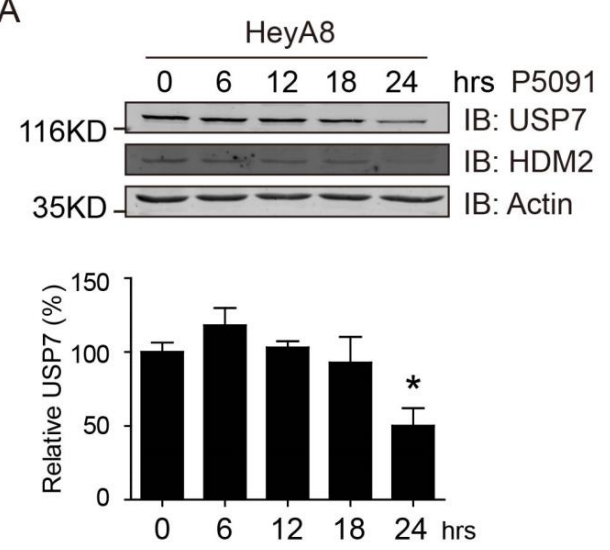

C

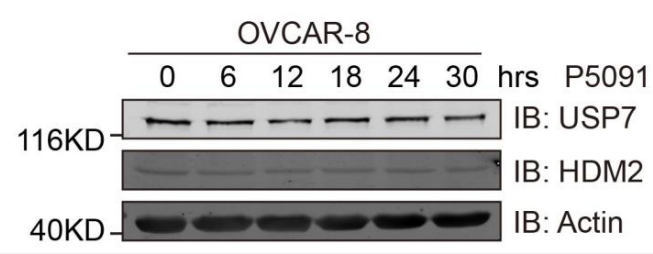

B
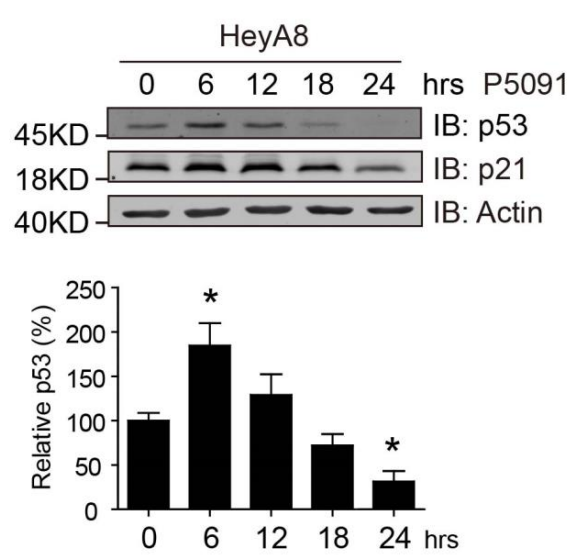

D

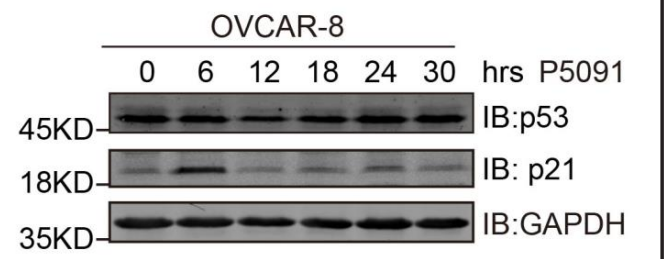

Fig. 4. P5091 regulates p53 and p21 levels through HDM2 in HeyA8 cells. A and B, HeyA8 cells were treated with P5091 for indicated times, and cell lysates were collected. Samples were analysed by immunoblotting using USP7, HDM2, p53, and p21 antibodies. Actin was probed to confirm equal loading. Bar charts below show quantification data from 3 independent experiments. All error bars represent standard error of the mean ( $\mathrm{n}=3$, * represents $\mathrm{p}<0.05$ ). C and D, OVCAR-8 cells were treated identically to HeyA8, and cell lysates were separated by SDS-PAGE followed by immunoblotting assays using USP7, HDM2, p53, p21, Actin, and GAPDH antibodies.

P5091 regulates HDM2 and p53 levels in HeyA8 cells

In the initial study on the USP7 inhibitor P5091, Anderson and colleagues investigated the effects of P5091 on multiple myeloma cells [22]. It was shown that P5091 destabilised HDM2, upregulated p53 and p21, and caused apoptosis. Therefore, we performed immunoblotting assays to investigate whether the same scenario occurred in ovarian cancer cells. In HeyA8 cells, the levels of HDM2 and USP7 decreased after P5091 treatment, most prominently at 24 hours (Fig. 4A). A concomitant upregulation in p53 and p21 expression was observed at 6 hours of P5091 treatment, followed by a gradual decrease (Fig. 4B). Conversely, USP7 remained relatively stable in OVCAR-8 cells until 30 hours of P5091 treatment, although slightly reduced HDM2 expression was observed (Fig. 4C). Furthermore, the abundance of p53 was not sensitive to P5091 exposure in OVCAR-8 cells despite the prolonged incubation time, although p21 showed an upregulation at 6 hours (Fig. 4D).

\section{P5091-induced cell death associated with autophagy}

In the attempt to understand the different responses to P5091 treatment of HeyA8 and OVCAR-8 cells, we observed that many vacuoles accumulated in P5091-treated OVCAR-8 cells but not in control cells or inhibitor-treated HeyA8 cells (Fig. 5A). This phenomenon prompted us to speculate that P5091 might induce autophagy in OVCAR-8 cells. Therefore, we performed immunoblotting experiments to examine the pivotal markers of autophagy, LC3 and p62. Interestingly, the LC3-I to LC3-II transition was enhanced by P5091 in both HeyA8 and OVCAR- 8 cells at 6 and 12 hours, indicating increased autophagosome formation (Fig. 5B). In HeyA8 cells, P5091 treatment led to significantly reduced p62 expression levels after 24 hours, while the inhibitor only caused a marginal decrease after prolonged 


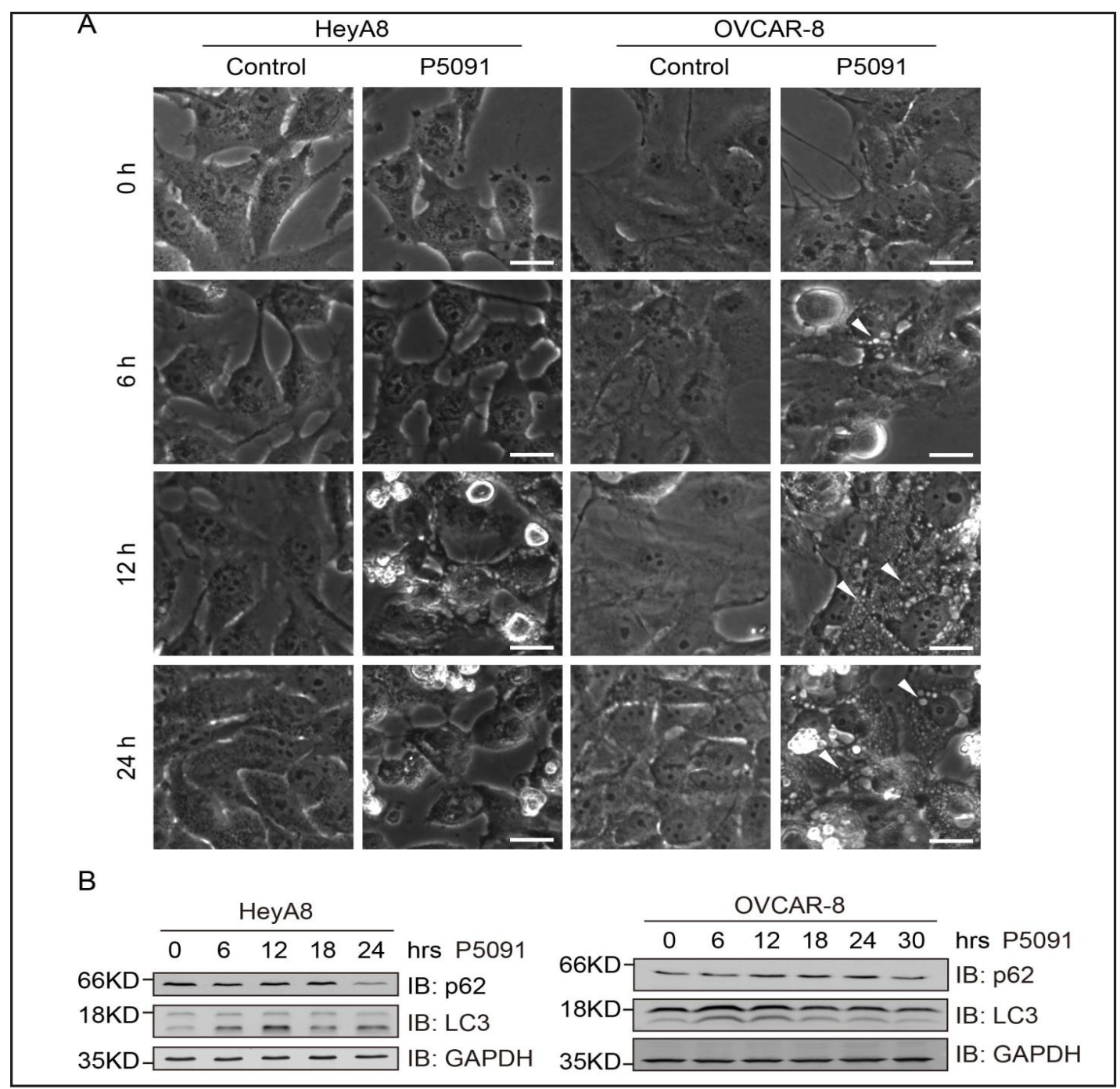

Fig. 5. P5091-induced cell death is accompanied by autophagy. A, HeyA8 and OVCAR-8 cells were treated with $50 \mu \mathrm{M}$ of $\mathrm{P} 5091$ for indicated times and examined under a phase contrast microscope at higher magnification (40X objective). Control cells were treated with DMSO. Intracellular vacuoles formed during P5091 exposure are evident in OVCAR-8 cells, with triangles pointing to representative examples. Scale bar $=25 \mu \mathrm{m}$. B, ovarian cancer cells were treated for indicated times as described in panel A, and cellular proteins were harvested for immunoblotting assays with p62 and LC3 antibodies. GAPDH was probed to show equal loading.

incubation (by 30 hours) with OVCAR-8 cells (Fig. 5B). These immunoblotting results confirm that P5091 can induce autophagy in both ovarian cancer cell lines as revealed by increased autophagosome formation, although the degradation of p62 was more efficient in HeyA8 cells compared to OVCAR-8 cells.

\section{Discussion}

Ovarian malignancy is a common type of cancer diagnosed in women [23-25]. Unfortunately, patients are often diagnosed at the later stages of the disease, thus facing limited therapeutic options and poor clinical outcomes [26]. Neoadjuvant therapy combining chemotherapy and surgery has proven effective and is frequently clinically administered [27, 
28]. Recent progressions in targeted therapy for ovarian cancer management involve PARP inhibitors exemplified by olaparib and rucaparib, which have shown promising results for patients carrying mutations in BRCA1 or BRCA2 [29, 30]. Nonetheless, BRCA1/2 mutationpositive cancers only compose less than $10 \%$ of all ovarian cancers, and the overall 5 year survival rate of ovarian cancer remains unsatisfactory [31,32]. To develop novel therapeutic strategies to improve ovarian cancer survival, there is urgent need to discover additional therapeutic target that can ideally be pharmacologically intervened [33, 34].

Recent investigations have linked the expression of deubiquitylase USP7 with poor prognosis in ovarian cancer $[16,35]$. Therefore, in the current study we investigated the effectiveness of the USP7 inhibitor P5091 to suppress ovarian cancer growth in vitro. P5091 was originally identified through a high-throughput screen as a specific inhibitor to USP7 with an $\mathrm{EC}_{50}$ of less than $5 \mu \mathrm{M}$ [22]. It was shown to be capable of inducing apoptosis and overcoming bortezomib resistance in multiple myeloma [22]. Further studies in other cancer types revealed that P5091 can down-regulate CCDC6, sensitize lung neuroendocrine cancer to PARP inhibitors, and deter Wnt signalling to repress colorectal tumour growth [36, 37].

In our proliferation assays, P5091 inhibited the growth of SKOV3, HeyA8, and OVCAR-8 cells. Among the 3 ovarian cancer cell lines, SKOV3 did not express p53 protein, while HeyA8 and OVCAR-8 expressed wild-type and mutant p53, respectively $[38,39]$. Considering the evidence that P5091 can regulate p53 levels and SKOV3 was the least sensitive to P5091, we chose HeyA8 and OVCAR-8 cells for further study. Based on cell morphology observations and Hoechst 33342 staining, we reasoned that P5091 induced cell death in HeyA8 and OVCAR-8. As P5091 was initially reported to induce apoptosis in multiple myeloma cells, we assessed its effect in ovarian cancer cells by flow cytometry with PI and Annexin V doublestaining. As expected, P5091 also induced apoptosis in HeyA8 and OVCAR-8 cells, although less prominently in OVCAR-8 cells as judged by the immunoblotting of apoptosis-related markers. In addition, data from flow cytometry analyses revealed an augmented necrotic population in P5091 treated samples, with more substantial increases in HeyA8 than in OVCAR-8 cells.

From the mechanistic aspect, P5091 in HeyA8 cells decreased HDM2 and USP7 levels, leading to the early upregulation of p53 and p21 with subsequent decrease, while both USP7 and p53 remained stable in OVCAR-8 during P5091 treatment. These results correlate well with the phenotypic observations that P5091 led to more dramatic damages in HeyA8 compared to OVCAR-8 cells. In the previous report, p53 upregulation was also apparent following P5091 addition but persisted much longer in multiple myeloma cells [22]. In HeyA8 cells, p53 and p21 appeared to be labile following P5091-induced increase, suggesting an alternative E3 ligase or degradation pathway rather than the involvement of HDM2 $[40,41]$. Considering the fact that HeyA8 and OVCAR-8 harbour wild-type and mutant p53, respectively, it is likely that the increased sensitivity of HeyA8 cells to P5091 may be somewhat attributed to wild-type p53. However, it should be noted that the inhibitory effects of P5091 can happen in a p53-independent manner as described by Anderson and colleagues, which is consistent with our data on SKOV3 cells, which lacked p53. Another interesting observation is that in both HeyA8 and OVCAR-8 cells, P5091-induced cell death is accompanied by autophagy, with less efficient p62 degradation and accumulated vacuoles in OVCAR-8 cells. It thus seems that more severe cell death induced by P5091 correlates with efficient autophagy as observed in HeyA8 cells, while the moderate toxicity of P5091 is associated with less effective autophagy in OVCAR-8 cells. Notably, it has been proposed that mutant p53 can function to suppress autophagy, which is consistent with our observations in OVCAR-8 cells that carry a p53 mutant [42]. In addition, it has been shown that certain ovarian cancer cells, including multiple OVCAR cancer cell lines, exhibit the multi-drugresistant (MDR) feature [43]. Therefore, the differences in MDR capabilities of ovarian cancer cells may also contribute to their differential sensitivity to the USP7 inhibitor P5091.

In the present study, we evaluated the effects of P5091 on ovarian cancer cell growth and death in vitro. Our data reveal that P5091 can effectively inhibit cell growth and induce both necrosis and apoptosis, which expands our current knowledge on this USP7 inhibitor.

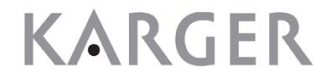




\section{Cellular Physiology Cell Physiol Biochem 2017;43:1755-1766

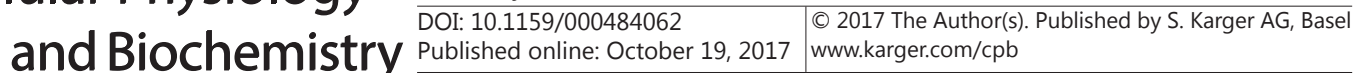 \\ Wang et al.: Targeting USP7 in Ovarian Cancer}

Furthermore, we showed that autophagy accompanied P5091-induced cell death in some case. However, limited ovarian cancer cell lines were used in this study, and a further inspection of other ovarian cancer cells with different p53 status is required to provide a better overview. In addition, the interplay between USP7 inhibition and p53-related autophagy awaits further investigation. To summarize, our observations provide evidence supporting the claim that USP7 can serve as a potential therapeutic target in ovarian cancer and thus warrant further studies.

\section{Acknowledgements}

Funding: LK and HL were supported by the National Natural Science Foundation of China [No. 81322029, No. 31371512, and No. 81301901]; HL was supported by the "Climbing Scholar" and "Excellent Talents" schemes of Liaoning Province, China. The authors are also grateful to Dr. Jin Q. Cheng (H. Lee Moffitt Cancer Center, US) for the T80 cell line.

\section{Disclosure Statement}

Competing financial interests: The authors declare no competing financial interests.

\section{References}

1 Mortality GBD, Causes of Death C: Global, regional, and national age-sex specific all-cause and causespecific mortality for 240 causes of death, 1990-2013: a systematic analysis for the Global Burden of Disease Study 2013. Lancet 2015;385:117-171.

$>2$ Li C, Ding H, Tian J, Wu L, Wang Y, Xing Y, Chen M: Forkhead Box Protein C2 (FOXC2) Promotes the Resistance of Human Ovarian Cancer Cells to Cisplatin In vitro and In vivo. Cell Physiol Biochem 2016;39:242-252.

-3 Chen Q Qin R, Fang Y, Li H: Berberine Sensitizes Human Ovarian Cancer Cells to Cisplatin Through miR-93/ PTEN/Akt Signaling Pathway. Cell Physiol Biochem 2015;36:956-965.

4 Kandoth C, McLellan MD, Vandin F, Ye K, Niu B, Lu C, Xie M, Zhang Q, McMichael JF, Wyczalkowski MA, Leiserson MD, Miller CA, Welch JS, Walter MJ, Wendl MC, Ley TJ, Wilson RK, Raphael BJ, Ding L: Mutational landscape and significance across 12 major cancer types. Nature 2013;502:333-339.

5 Crafton SM, Bixel K, Hays JL: PARP inhibition and gynecologic malignancies: A review of current literature and on-going trials. Gynecol Oncol 2016;10.1016/j.ygyno.2016.05.003

6 Chase DM, Chaplin DJ, Monk BJ: The development and use of vascular targeted therapy in ovarian cancer. Gynecol Oncol 2017;10.1016/j.ygyno.2017.01.031

7 Colombo N, Conte PF, Pignata S, Raspagliesi F, Scambia G: Bevacizumab in ovarian cancer: Focus on clinical data and future perspectives. Crit Rev Oncol Hematol 2016;97:335-348.

-8 Staropoli N, Ciliberto D, Chiellino S, Caglioti F, Giudice TD, Gualtieri S, Salvino A, Strangio A, Botta C, Pignata $\mathrm{S}$, Tassone P, Tagliaferri P: Is ovarian cancer a targetable disease? A systematic review and meta-analysis and genomic data investigation. Oncotarget 2016;7:82741-82756.

$>9$ Clague MJ, Heride C, Urbe S: The demographics of the ubiquitin system. Trends Cell Biol 2015;25:417-426.

10 Heride C, Urbe S, Clague MJ: Ubiquitin code assembly and disassembly. Curr Biol 2014;24:R215-220.

-11 Clague MJ, Barsukov I, Coulson JM, Liu H, Rigden DJ, Urbe S: Deubiquitylases from genes to organism. Physiol Rev 2013;93:1289-1315.

12 Komander D, Clague MJ, Urbe S: Breaking the chains: structure and function of the deubiquitinases. Nat Rev Mol Cell Biol 2009;10:550-563.

13 Nicholson B, Suresh Kumar KG: The multifaceted roles of USP7: new therapeutic opportunities. Cell Biochem Biophys 2011;60:61-68.

14 Sacco JJ, Coulson JM, Clague MJ, Urbe S: Emerging roles of deubiquitinases in cancer-associated pathways. IUBMB Life 2010;62:140-157. 


\section{Cellular Physiology Cell Physiol Biochem 2017;43:1755-1766

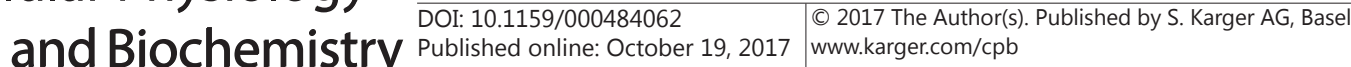

Wang et al.: Targeting USP7 in Ovarian Cancer

15 Ma M, Yu N: Ubiquitin-specific protease 7 expression is a prognostic factor in epithelial ovarian cancer and correlates with lymph node metastasis. Onco Targets Ther 2016;9:1559-1569.

16 Zhang L, Wang H, Tian L, Li H: Expression of USP7 and MARCH7 Is Correlated with Poor Prognosis in Epithelial Ovarian Cancer. Tohoku J Exp Med 2016;239:165-175.

17 Zhang Y, Zhang J, Liu C, Du S, Feng L, Luan X, Zhang Y, Shi Y, Wang T, Wu Y, Cheng W, Meng S, Li M, Liu H: Neratinib induces ErbB2 ubiquitylation and endocytic degradation via HSP90 dissociation in breast cancer cells. Cancer Lett 2016;382:176-185.

18 Khan MN, Wang B, Wei J, Zhang Y, Li Q, Luan X, Cheng JW, Gordon JR, Li F, Liu H: CXCR1/2 antagonism with CXCL8/Interleukin-8 analogue CXCL8(3-72)K11R/G31P restricts lung cancer growth by inhibiting tumor cell proliferation and suppressing angiogenesis. Oncotarget 2015;6:21315-21327.

19 Tian K, Zhong W, Zheng X, Zhang J, Liu P, Zhang W, Liu H: Neuroleukin/Autocrine Motility Factor Receptor Pathway Promotes Proliferation of Articular Chondrocytes through Activation of AKT and Smad2/3. Sci Rep 2015;5:15101.

20 Li P, Sun Y, Liu Q: MicroRNA-340 Induces Apoptosis and Inhibits Metastasis of Ovarian Cancer Cells by Inactivation of NF-x03BA;B1. Cell Physiol Biochem 2016;38:1915-1927.

-21 Son DS, Kabir SM, Dong YL, Lee E, Adunyah SE: Inhibitory effect of tumor suppressor p53 on proinflammatory chemokine expression in ovarian cancer cells by reducing proteasomal degradation of IkappaB. PLoS One 2012;7:e51116.

-22 Chauhan D, Tian Z, Nicholson B, Kumar KG, Zhou B, Carrasco R, McDermott JL, Leach CA, Fulcinniti M, Kodrasov MP, Weinstock J, Kingsbury WD, Hideshima T, Shah PK, Minvielle S, Altun M, Kessler BM, Orlowski R, Richardson P, Munshi N, Anderson KC: A small molecule inhibitor of ubiquitin-specific protease-7 induces apoptosis in multiple myeloma cells and overcomes bortezomib resistance. Cancer Cell 2012;22:345-358.

-23 Siegel RL, Miller KD, Jemal A: Cancer statistics, 2015. CA Cancer J Clin 2015;65:5-29. Siegel RL, Miller KD, Jemal A: Cancer statistics, 2016. CA Cancer J Clin 2016;66:7-30. Siegel RL, Miller KD, Jemal A: Cancer Statistics, 2017. CA Cancer J Clin 2017;67:7-30. Grunewald T, Ledermann JA: Targeted Therapies for Ovarian Cancer. Best Pract Res Clin Obstet Gynaecol 2016;10.1016/j.bpobgyn.2016.12.001

27 Hall TR, Dizon DS: Neoadjuvant chemotherapy for advanced epithelial ovarian cancer. Clin Adv Hematol Oncol 2016;14:262-268.

28 Wright AA, Bohlke K, Armstrong DK, Bookman MA, Cliby WA, Coleman RL, Dizon DS, Kash JJ, Meyer LA, Moore KN, Olawaiye AB, Oldham J, Salani R, Sparacio D, Tew WP, Vergote I, Edelson MI: Neoadjuvant Chemotherapy for Newly Diagnosed, Advanced Ovarian Cancer: Society of Gynecologic Oncology and American Society of Clinical Oncology Clinical Practice Guideline. J Clin Oncol 2016;34:3460-3473.

29 Lin KY, Kraus WL: PARP Inhibitors for Cancer Therapy. Cell 2017;169:183.

-30 Dulaney C, Marcrom S, Stanley J, Yang ES: Poly(ADP-ribose) polymerase activity and inhibition in cancer. Semin Cell Dev Biol 2017;63:144-153.

-31 Miller KD, Siegel RL, Lin CC, Mariotto AB, Kramer JL, Rowland JH, Stein KD, Alteri R, Jemal A: Cancer treatment and survivorship statistics, 2016. CA Cancer J Clin 2016;66:271-289.

-32 Li Y, Li Y, Zhang J, Zheng C, Zhu H, Yu H, Fan L: Circulating Insulin-Like Growth Factor-1 Level and Ovarian Cancer Risk. Cell Physiol Biochem 2016;38:589-597.

-33 Deng Y, Wang Z, Zhang F, Qiao M, Yan Z, Wei Q Wang J, Liu H, Fan J, Zou Y, Liao J, Hu X, Chen L, Yu X, Haydon RC, Luu HH, Qi H, He TC, Zhang J: A Blockade of IGF Signaling Sensitizes Human Ovarian Cancer Cells to the Anthelmintic Niclosamide-Induced Anti-Proliferative and Anticancer Activities. Cell Physiol Biochem 2016;39:871-888.

34 Pelzl L, Hosseinzadeh Z, Alzoubi K, Al-Maghout T, Schmidt S, Stournaras C, Lang F: Impact of Na+/Ca2+ Exchangers on Therapy Resistance of Ovary Carcinoma Cells. Cell Physiol Biochem 2015;37:1857-1868.

35 Qin D, Wang W, Lei H, Luo H, Cai H, Tang C, Wu Y, Wang Y, Jin J, Xiao W, Wang T, Ma C, Xu H, Zhang J, Gao F, Wu YL: CDDO-Me reveals USP7 as a novel target in ovarian cancer cells. Oncotarget 2016;7:77096-77109.

-36 An T, Gong Y, Li X, Kong L, Ma P, Gong L, Zhu H, Yu C, Liu J, Zhou H, Mao B, Li Y: USP7 inhibitor P5091 inhibits Wnt signaling and colorectal tumor growth. Biochem Pharmacol 2017;131:29-39.

37 Malapelle U, Morra F, Ilardi G, Visconti R, Merolla F, Cerrato A, Napolitano V, Monaco R, Guggino G, Monaco G, Staibano S, Troncone G, Celetti A: USP7 inhibitors, downregulating CCDC6, sensitize lung neuroendocrine cancer cells to PARP-inhibitor drugs. Lung Cancer 2017;107:41-49. 


\section{Cellular Physiology Cell Physiol Biochem 2017;43:1755-1766 and Biochemistry Published 10/159/000484062, 2017 O 2017 The Author(s). Published by S. Karger AG, Basel}

Wang et al.: Targeting USP7 in Ovarian Cancer

-38 O’Connor PM, Jackman J, Bae I, Myers TG, Fan S, Mutoh M, Scudiero DA, Monks A, Sausville EA, Weinstein JN, Friend S, Fornace AJ, Jr., Kohn KW: Characterization of the p53 tumor suppressor pathway in cell lines of the National Cancer Institute anticancer drug screen and correlations with the growth-inhibitory potency of 123 anticancer agents. Cancer Res 1997;57:4285-4300.

-39 Leroy B, Fournier JL, Ishioka C, Monti P, Inga A, Fronza G, Soussi T: The TP53 website: an integrative resource centre for the TP53 mutation database and TP53 mutant analysis. Nucleic Acids Res 2013;41:D962-969.

40 Sane S, Rezvani K: Essential Roles of E3 Ubiquitin Ligases in p53 Regulation. Int J Mol Sci 2017;18:

41 Lee JT, Gu W: The multiple levels of regulation by p53 ubiquitination. Cell Death Differ 2010;17:86-92.

42 Cordani M, Butera G, Pacchiana R, Donadelli M: Molecular interplay between mutant p53 proteins and autophagy in cancer cells. Biochim Biophys Acta 2017;1867:19-28.

43 Roberts D, Schick J, Conway S, Biade S, Laub PB, Stevenson JP, Hamilton TC, O’Dwyer PJ, Johnson SW: Identification of genes associated with platinum drug sensitivity and resistance in human ovarian cancer cells. Br J Cancer 2005;92:1149-1158. 